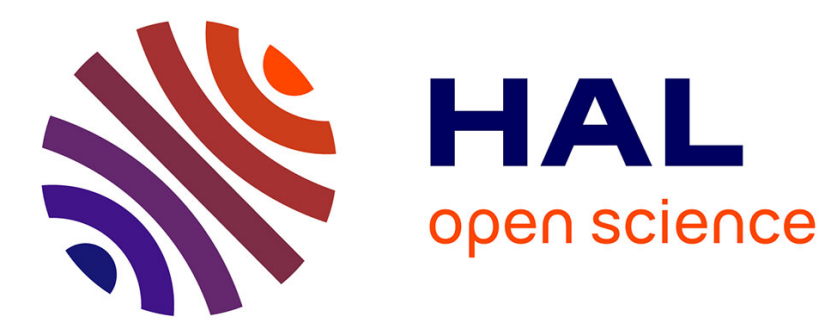

\title{
On the existence of positive solutions to semilinear elliptic systems involving gradient term
}

Boumediene Abdellaoui, Ahmed Attar, El-Haj Laamri

\section{To cite this version:}

Boumediene Abdellaoui, Ahmed Attar, El-Haj Laamri. On the existence of positive solutions to semilinear elliptic systems involving gradient term. Applicable Analysis, 2018, 10.1080/00036811.2017.1419204. hal-01748590

\section{HAL Id: hal-01748590 \\ https://hal.science/hal-01748590}

Submitted on 29 Mar 2018

HAL is a multi-disciplinary open access archive for the deposit and dissemination of scientific research documents, whether they are published or not. The documents may come from teaching and research institutions in France or abroad, or from public or private research centers.
L'archive ouverte pluridisciplinaire HAL, est destinée au dépôt et à la diffusion de documents scientifiques de niveau recherche, publiés ou non, émanant des établissements d'enseignement et de recherche français ou étrangers, des laboratoires publics ou privés. 


\title{
ON THE EXISTENCE OF POSITIVE SOLUTIONS TO SEMILINEAR ELLIPTIC SYSTEMS INVOLVING GRADIENT TERM
}

\author{
BOUMEDIENE ABDELLAOUI, AHMED ATTAR, EL-HAJ LAAMRI
}

Abstract. In this work we analyze the existence of solutions to the nonlinear elliptic system:

$$
\left\{\begin{aligned}
-\Delta u & =v^{q}+\alpha g & & \text { in } \Omega, \\
-\Delta v & =|\nabla u|^{p}+\lambda f & & \text { in } \Omega, \\
u=v & =0 & & \text { on } \partial \Omega, \\
u, v & \geq 0 & & \text { in } \Omega,
\end{aligned}\right.
$$

where $\Omega$ is a bounded domain of $\mathbb{R}^{N}$ and $p \geq 1, q>0$ with $p q>1$. $f, g$ are nonnegative measurable functions with additional hypotheses and $\alpha, \lambda \geq 0$.

As a consequence we show that the fourth order problem

$$
\left\{\begin{aligned}
\Delta^{2} u & =|\nabla u|^{p}+\tilde{\lambda} \tilde{f} & & \text { in } \Omega, \\
u=\Delta u & =0 & & \text { on } \partial \Omega,
\end{aligned}\right.
$$

has a solution for all $p>1$, under suitable conditions on $\tilde{f}$ and $\tilde{\lambda}$.

\section{INTRODUCTION}

The aim of this paper is to discuss the existence of solutions to the following elliptic system

$$
\left\{\begin{aligned}
-\Delta u & =v^{q}+\alpha g & & \text { in } \Omega, \\
-\Delta v & =|\nabla u|^{p}+\lambda f & & \text { in } \Omega, \\
u=v & =0 & & \text { on } \partial \Omega, \\
u, v & \geq 0 & & \text { in } \Omega,
\end{aligned}\right.
$$

where $\Omega \subset \mathbb{R}^{N}$ is a bounded domain. We will consider the case $p \geq 1, q>0$ with $p q>1, f, g$ are nonnegative measurable functions and $\alpha, \lambda$ are nonnegative real constants.

Our goal is to get natural conditions on the parameter $\alpha, \lambda$ and the data $f, g$, in order to prove the existence of positive solutions to the problem (1.1) under the condition $p q>1$. By solution, we mean solution in the sense of distributions (see Definition 2.1).

The class of elliptic systems with gradient term appears when considering electrochemical models in engineering and some other model in fluid dynamics. We refer to [15], and [16] for more details and more applications of this class of systems.

Existence results for nonlinear elliptic systems with gradient term are well known in some particular cases. For example, in the case where $\alpha=\lambda=1$, the authors in [4] established that

Date: January 3, 2018.

Key words and phrases. Elliptic System, nonlinear gradient terms, Bi-Laplacien operator.

2000 Mathematics Subject Classification:MSC 2000:35J55, 35D10, 35J60,

The first author is partially supported by project MTM2016-80474-P, MINECO, Spain. 
system (1.1) has a solution for all $(f, g) \in L^{2}(\Omega) \times L^{2}(\Omega)$ under the condition that $p q<1$ and $0<p<2$. In [11], Boccardo-Orsina-Porretta investigated the system

$$
\left\{\begin{aligned}
-\operatorname{div}(b(x, z) \nabla u) & =f(x) & & \text { in } \Omega, \\
-\operatorname{div}(a(x, z) \nabla z) & =b(x, z)|\nabla u|^{2} & & \text { in } \Omega, \\
u=z & =0 & & \text { on } \partial \Omega,
\end{aligned}\right.
$$

where the functions $(x, s) \mapsto a(x, s), b(x, s)$ are positive and coercive Carathéodory functions. Under the hypothesis that $f \in L^{m}(\Omega)$, with $m \geq \frac{2 N}{N+2}$, they proved the existence and the regularity of a positive solution. In [12], Boccardo-Orsina-Puel studied the system

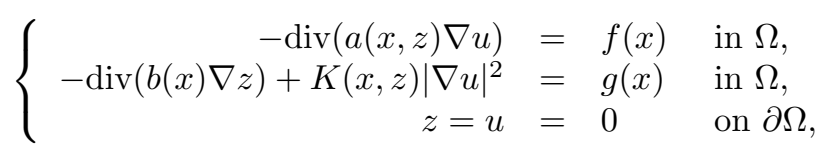

where the gradient appears as an absorption term.

It is clear that for $g=0$, setting $v=|-\Delta u|^{\frac{1}{q}-1}(-\Delta u)$, the system (1.1) is reduced to the following fourth order problem

$$
\left\{\begin{aligned}
-\Delta\left(|-\Delta u|^{\frac{1}{q}-1}(-\Delta u)\right) & =|\nabla u|^{p}+\lambda f & & \text { in } \Omega, \\
u=\Delta u & =0 & & \text { on } \partial \Omega, \\
u & >0 & & \text { in } \Omega .
\end{aligned}\right.
$$

If $q=1$, the above problem is reduced to the following one

$$
\left\{\begin{aligned}
\Delta^{2} u & =|\nabla u|^{p}+\lambda f & & \text { in } \Omega, \\
u=\Delta u & =0 & & \text { on } \partial \Omega, \\
u & \geq 0 & & \text { in } \Omega .
\end{aligned}\right.
$$

Hence, to get a positive solution to problem (1.3), we just have to show that the system (1.1) has a solution with $q=1$ and $\alpha=0$.

Problems related to the Bi-Laplacian operator are widely studied in the literature, we refer to [19] and the references therein. We refer also to the paper [17] where an extension of the Kardar-ParisiZhang equation for the Bi-Laplacian operator is also studied.

Notice that if we substitute the fourth order operator $\Delta^{2} u$ by the classical Laplacian, the previous problem takes the form

$$
\left\{\begin{aligned}
-\Delta u & =|\nabla u|^{p}+\lambda f & & \text { in } \Omega \\
u & =0 & & \text { on } \partial \Omega .
\end{aligned}\right.
$$

This problem has been widely studied in the literature, we refer to $[23,10,9,14,18,7,21,24]$ and references therein. If $p \leq 2$, using suitable comparison principle and under suitable hypothesis on $f$, Alaa-Pierre proved in [3] the existence of positive solutions to (1.4) that is in a suitable Sobolev space. Some extension were proved in [20] using truncations arguments. The existence result in [20] holds for a more general class of elliptic operators. However, in the case of the Laplacian operator, the condition $p \leq 2$ seems to be optimal and cannot be improved by using the techniques of [20]. On the other hand, if $u \in W_{0}^{1,2}(\Omega)$, then in general, $\Delta\left(T_{k}(u)\right) \notin L^{1}(\Omega)$ and it seems to be more complicated to handle problem (1.3) using truncation arguments. The existence result obtained in [22] is more general because it holds for all $p>1$. However, it seems that the techniques of the proofs are strongly related to a fine estimate on the Green function and cannot be extended to 
more general operators.

In the case where $\Omega=B_{R}(0)$, then for $p=1, q=2$ and $g=f=0$, based on ODEs technics, the authors in [16] were able to show that the corresponding system has a unique radial large solution.

Our paper mainly complements the investigations of [4]. However as it was noticed in [4], the arguments used to deal with the case $p q<1$ can not be adapted to the case $p q>1$. Some new arguments are needed to deal with the new situation. Hence, we will use in a convenient way, the Schauder fixed point theorem, following some ideas from Phuc in [26]. Moreover, in order to show that (1.1) has a solution, it is necessary to have regularity assumptions on the data $f, g$ and smallness conditions on $\lambda$ and $\alpha$. Indeed, such conditions are necessary even in the case of a single equation. To help understand the situation, let us consider the following semilinear equation :

$$
\left(P_{r}\right)\left\{\begin{aligned}
-\Delta w & =w^{r}+h & & \text { in } \Omega, \\
w & =0 & & \text { on } \partial \Omega, \\
w & \geq 0 & & \text { in } \Omega,
\end{aligned}\right.
$$

where $r \in(1,+\infty)$ and $h \geq 0$. As it is proved by Baras-Pierre in [5], two conditions on $h$ are necessary for the existence of solutions to $\left(P_{r}\right)$ :

(i) A regularity condition : $h$ should be "regular" enough.

(ii) A size condition : even if $h \in \mathcal{C}_{0}^{\infty}(\Omega)$, it should be small enough. For instance, if $h=\gamma \psi$ where $\psi \in \mathcal{C}_{0}^{\infty}(\Omega), \psi \supsetneqq 0$ and $\gamma>0$, then there exists $\gamma^{*}>0$ so that $\left(P_{r}\right)$ does not have any solution for $\gamma>\gamma^{*}$.

In other words, a necessary and sufficient condition on $h$ for the existence of solutions to $\left(P_{r}\right)$ can be formulated in a simple way, saying that a certain "norm" of $h$ should be exactly less than or equal to $k(r)=\frac{r-1}{r^{r^{\prime}}}$ where $r^{\prime}=\frac{r}{r-1}$. This quantity is defined by duality through a functional which is naturally associated with the above problem. Mathematically speaking, one has the following result :

Theorem 1.1 (Baras-Pierre [5]). Let $h$ be a nonnegative measurable function on $\Omega$. Then $\left(P_{r}\right)$ has a weak solution if and only if

$$
(H)\left\{\begin{array}{l}
\forall \varphi \in \mathcal{C}^{\infty}(\Omega) \text { with } \varphi \supsetneqq 0 \text { in } \Omega \text { and } \varphi=0 \text { on } \partial \Omega, \\
\int_{\Omega} h \varphi \leq k(r) \int_{\Omega}|\Delta \varphi|^{r^{\prime}}|\varphi|^{1-r^{\prime}} .
\end{array}\right.
$$

Thus, we will deal with this type of functionals in order to describe exactly the optimal size of the data $f, g$ and to determine the largest set of $(\lambda, \alpha)$ such that the system (1.1) has a solution.

The paper is divided into four sections. We state our main results in section 2 . Then we introduce some useful tools in section 3. Finally the last section is devoted to prove our theorems and we also give some nonexistence results that show, in some sense, the optimality of the hypothesis imposed in Theorem 2.4 . 


\section{Main RESUlts}

Throughout this paper, $\Omega$ is a bounded open set of $\mathbb{R}^{N}$ and $f, g: \Omega \rightarrow \mathbb{R}$ are nonnegative measurable functions.

Let's begin by specifying the meaning of a solution.

Definition 2.1. Assume that $p \geq 1$ and $q>0$. Let $f, g$ be nonnegative functions such that $f, g \in L^{1}(\Omega)$. We say that $(u, v) \in L^{1}(\Omega) \times L^{1}(\Omega)$, with $u, v \geq 0$, is a weak solution to system (1.1) if $(u, v) \in W_{0}^{1, p}(\Omega) \times W_{0}^{1,1}(\Omega), v^{q} \in L^{1}(\Omega)$ and for all $\varphi, \psi \in \mathcal{C}_{0}^{\infty}(\Omega)$, we have

$$
\int_{\Omega} \nabla \varphi \nabla u=\int_{\Omega} v^{q} \varphi+\alpha \int_{\Omega} g \varphi, \text { and } \int_{\Omega} \nabla \psi \nabla v=\int_{\Omega}|\nabla u|^{p} \psi+\lambda \int_{\Omega} f \psi .
$$

We are now ready to state our main existence results.

Theorem 2.2. Assume that $p \geq 1, q>0$ with $p q>1$. Let $(m, \sigma) \in[1,+\infty)^{2}$. Suppose that $(f, g) \in L^{m}(\Omega) \times L^{\sigma}(\Omega)$ where $(m, \sigma)$ satisfies one of the following conditions

$$
\left\{\begin{aligned}
m, \sigma & \in[1, N), \\
p m & <\frac{\sigma N}{N-\sigma}=\sigma^{*}, \\
\frac{q \sigma}{N+q \sigma} & <\frac{m}{N-m},
\end{aligned}\right.
$$

or

$$
m \geq N \text { and } \sigma>\frac{p m N}{N+p m}
$$

or

$$
\sigma \geq N \text { and } m>\frac{q \sigma N}{N+2 q \sigma} \text {. }
$$

Then there exists $\Lambda^{*}>0$ such that for all $(\lambda, \alpha) \in \Pi$ where

$$
\Pi:=\left\{(\lambda, \alpha) \in[0,+\infty) \times[0,+\infty) \mid \lambda\|f\|_{m}+\alpha^{p}\|g\|_{\sigma}^{p} \leq \Lambda^{*}\right\},
$$

the system (1.1) has a nonnegative solution $(u, v)$. Moreover $(u, v) \in W_{0}^{1, \theta}(\Omega) \times W_{0}^{1, r}(\Omega)$ for all $\theta<\frac{\sigma N}{(N-\sigma)_{+}}$and $r<\frac{m N}{(N-m)_{+}}$.

\section{Remark 1.}

(1) To give some light on the hypothesis (2.2), (2.3) and (2.4), let us make explicit the size conditions on $(p, q)$ for a given $(m, \sigma)$.

(i) If $m=\sigma=1$ and $N \geq 2$, then (2.2) is satisfied for $p<\frac{N}{N-1}$ and $q<\frac{N}{(N-2)_{+}}$, that is the classical range when dealing with entropy solution.

(ii) $m=\sigma=2$ and $N \geq 3$, then (2.2) is satisfied for $p<\frac{N}{N-1}$ and $q<\frac{N}{N-2}$. 
(iii) $m=\sigma=N / 2$ and $N \geq 3$, then (2.2) is satisfied for $p<2$ and for all $q$.

(iv) $m=\sigma \geq N$, then (2.3) and (2.4) are satisfied for all $(p, q) \in[1,+\infty)^{2}$.

(v) $\sigma=N$ and $m=N / 2$, then (2.4) are satisfied for all $(p, q) \in[1,+\infty)^{2}$.

(vi) $m=N$ and $\sigma=\frac{3 N}{4}$, then (2.3) is satisfied for all $p<3$ and all $q$.

(2) Reciprocally,

(i) If $p<\frac{N}{N-1}$ and $q<\frac{N}{N-2}$, then (2.2) holds for all $\sigma, m \geq 1$.

(ii) If $p \geq \frac{N}{N-1}$ or $q \geq \frac{N}{N-2}$, then $(\sigma, m)$ satisfies

$$
\left\{\begin{aligned}
m, \sigma & \geq 1 \\
\frac{1}{q \sigma}+\frac{2}{N} & >\frac{1}{m} \\
\frac{1}{p m}+\frac{1}{N} & >\frac{1}{\sigma}
\end{aligned}\right.
$$

It is clear that the above condition hold always if we choose $m>\frac{N}{2}$ and $\sigma>N$ independently of the values of $p$ and $q$.

Notice that the set $\Pi$ defined in $(2.5)$ is a bounded set of $\mathbb{R}_{+}^{2}$. The next nonexistence result explains clearly that a smallness condition on $(\lambda, \alpha)$ is necessary for the existence of solutions to (1.1), at least for $p>1$ and $q \geq 1$.

Theorem 2.3. Suppose that $p>1$ and $q \geq 1$. Let $f, g$ be nonnegative measurable functions such that $(f, g) \neq(0,0)$ and $f, g \in L^{1}(\Omega)$. Let $(\lambda, \alpha) \in(0,+\infty)^{2}$ be such that the system (1.1) has a nonnegative solution $(u, v)$, then there exists $\left(\lambda^{*}, \alpha^{*}\right) \in(0,+\infty)^{2}$ such that $\lambda \leq \lambda^{*}$ and $\alpha \leq \alpha^{*}$.

In the particular case where $q=1$ and $\alpha \equiv 0$, and as a direct application of Theorem 2.2, we obtain the following existence result for the Bi-Laplacian problem with gradient term.

Theorem 2.4. Let $p>1$. Suppose $0 \supsetneqq f \in L^{m}(\Omega)$ where $m>\max \left\{1, \frac{N}{3 p^{\prime}}\right\}$. Then there exists $\lambda^{*}>0$ such that if $\lambda<\lambda^{*}$ the problem

$$
\left\{\begin{aligned}
\Delta^{2} u & =|\nabla u|^{p}+\lambda f & & \text { in } \Omega, \\
u=\Delta u & =0 & & \text { on } \partial \Omega, \\
u & \geq 0 & & \text { in } \Omega .
\end{aligned}\right.
$$

has a solution $u$ such that $u \in W^{4, m}(\Omega)$ if $m<\frac{N}{2}$ and $u \in \mathcal{C}^{s}(\Omega)$ with $s<4-\frac{N}{m}$ if $m>\frac{N}{2}$.

Finally we give a nonexistence result that, in some sense, justifies the regularity conditions imposed on $f$ to get the existence of nonnegative solution to problem (2.7).

Theorem 2.5. Assume that $1 \leq m<\max \left\{1, \frac{N}{3 p^{\prime}}\right\}$ where $p>1$. Then there exists $f \in L^{m}(\Omega)$ with $f \gtrless 0$ such that problem (2.7) does not have any positive solution for all $\lambda>0$. 


\section{UsEFul RESUlts}

For the convenience of the reader and for the sake of completeness, we recall in this short section some classical results we will use in our proofs.

In order to prove the main existence result of this paper we will use useful theorems: Schauder fixed point Theorem and Vitali's Theorem.

Theorem 3.1 (Schauder fixed point theorem). Assume that $E$ is a closed convex set of a Banach space $X$. Let $T$ be a continuous and compact mapping from $E$ into itself. Then $T$ has a fixed point in $E$.

Theorem 3.2. (Vitali) Let $(E, \mu)$ be a measured space such that $\mu(E)<+\infty$, let $1 \leq p<+\infty$ and let $\left\{f_{n}\right\}_{n} \subset L^{p}(E)$ such that $f_{n} \rightarrow f$ a.e. If $\left\{f_{n}^{p}\right\}_{n}$ is uniformly integrable over $E$, then $f \in L^{p}(E)$ and $f_{n} \rightarrow f$ in $L^{p}(E)$.

Systematically, we will use the following regularity result proved in [6, Appendix].

Theorem 3.3. Assume that $h \in L^{1}(\Omega)$, then the problem

$$
\left\{\begin{array}{rlll}
-\Delta z & = & & \text { in } \Omega, \\
z & = & & \text { on } \partial \Omega
\end{array}\right.
$$

has a unique weak solution $z \in W_{0}^{1, s}(\Omega)$ for all $s \in\left[1, \frac{N}{N-1}\right)$.

Moreover, for $s \in\left[1, \frac{N}{N-1}\right)$ fixed, there exists a positive constant $C=C(\Omega, N, s)$ such that

$$
\|\nabla z\|_{L^{s}(\Omega)} \leq C\|h\|_{L^{1}(\Omega)} .
$$

and the operator $\Phi: h \mapsto z$ is compact from $L^{1}(\Omega)$ into $W_{0}^{1, s}(\Omega)$.

Finally, let us recall the following classical regularity result that we will use in several proofs below.

Theorem 3.4. Let $h \in L^{m}(\Omega)$ with $m>1$. Then the problem

$$
\left\{\begin{array}{rll}
-\Delta z & = & \text { in } \Omega \\
z= & 0 & \text { on } \partial \Omega .
\end{array}\right.
$$

has a unique weak solution $z$. Moreover there exists a positive constant $C=C(\Omega, N, m)$ independent of $h$ such that

(1) If $1<m<N$, then

$$
\|\nabla z\|_{L^{m^{*}}(\Omega)} \leq C|| h \|_{L^{m}(\Omega)} \text { where } m^{*}=\frac{m N}{N-m} .
$$

(2) If $m=N,|\nabla z| \in L^{s}(\Omega)$ for all $s \in[1,+\infty)$.

(3) If $m>N, z \in \mathcal{C}^{1, \gamma}(\Omega)$ for some $\gamma \in(0,1)$.

For a proof see for instance [13] or [8].

Remark 2. As a consequence of the above theorems, we can prove that for all $h \in L^{1}(\Omega)$, there exists a unique solution $w$ of the problem

$$
\left\{\begin{array}{rlll}
\Delta^{2} w & = & h & \text { in } \Omega, \\
w=\Delta w & = & 0 & \text { on } \partial \Omega,
\end{array}\right.
$$


with $w \in W_{0}^{1, \theta}(\Omega)$ for all $\theta<\frac{N}{N-3}$ and

$$
\|w\|_{W_{0}^{1, \theta}(\Omega)} \leq C(\Omega, \theta)\|h\|_{L^{1}(\Omega)} .
$$

\section{Proofs of the main Results.}

4.1. Proof of Theorem 2.2. We give the proof in the case where $(m, \sigma)$ satisfies $(2.2)$ i.e.

$$
m, \sigma \in[1, N), p m<\frac{\sigma N}{N-\sigma}=\sigma^{*}, \frac{q \sigma}{N+q \sigma}<\frac{m}{N-m} .
$$

The other cases follow by using the same arguments.

For $s \geq 0$, we define the function

$$
\Upsilon(s)=s^{\frac{1}{p q}}-\tilde{C} s,
$$

where $\tilde{C}$ is a universal positive constant that depends only on datum and that will specified later. Using the fact that $p q>1$, then there exists $s_{0}>0$ such that $\Upsilon\left(s_{0}\right)=0, \Upsilon(s)>0, \forall s \in\left(0, s_{0}\right)$, $\Upsilon(s)<0, \forall s \in\left(s_{0},+\infty\right)$ and we get the existence of positive constants $\ell>0$ and $\hat{\Lambda}>0$ such that

$$
\max _{s \geq 0} \Upsilon(s)=\Upsilon(\ell)=\hat{\Lambda} \text {. }
$$

Thus

$$
\ell^{\frac{1}{p q}}=\tilde{C}\left(\ell+\frac{\hat{\Lambda}}{\tilde{C}}\right)
$$

Fix $\ell>0$ as above and define the set

$$
\Pi \equiv\left\{(\lambda, \alpha) \in[0,+\infty) \times[0,+\infty) ; \lambda\|f\|_{L^{m}(\Omega)}+\alpha^{p}\|g\|_{L^{\sigma}(\Omega)}^{p} \leq \frac{\hat{\Lambda}}{\tilde{C}} \equiv \Lambda^{*}\right\} .
$$

It is clear that $\Pi$ is non empty, bounded and for all $(\lambda, \alpha) \in \Pi$, we have

$$
\tilde{C}\left(\ell+\lambda\|f\|_{L^{m}(\Omega)}+\alpha^{p}\|g\|_{L^{\sigma}(\Omega)}^{p}\right) \leq \ell^{\frac{1}{p^{q}}} .
$$

In what follows we fix $(\lambda, \alpha) \in \Pi$. Since $\frac{q \sigma N}{N+q \sigma}<\frac{m N}{N-m}$, there exists $r>1$ such that

$$
\frac{q \sigma N}{N+q \sigma}<r<\frac{m N}{N-m} \text {. }
$$

Moreover, if $r<N$, then

$$
\sigma q<\frac{r N}{N-r}=r^{*}
$$

and if $r \geq N$, then (4.3) holds trivially with $r^{*}=\infty$.

Now let us fix $\ell$ and $r$ as above, we define the set

$$
E=\left\{w \in W_{0}^{1,1}(\Omega) ; w \in W_{0}^{1, r}(\Omega) \text { and }\|\nabla w\|_{L^{r}(\Omega)} \leq \ell^{\frac{1}{p q}}\right\} .
$$

One can easily verify that $E$ is a closed convex subset of $W_{0}^{1,1}(\Omega)$. Let us consider the operator

$$
\begin{gathered}
T: E \longrightarrow W_{0}^{1,1}(\Omega) \\
w \longmapsto T(w)=v
\end{gathered}
$$


where $v$ is the unique solution to problem

$$
\left\{\begin{aligned}
-\Delta v & =|\nabla u|^{p}+\lambda f & & \text { in } \Omega \\
v & =0 & & \text { on } \partial \Omega,
\end{aligned}\right.
$$

with $u$ being the unique solution to the problem

$$
\left\{\begin{aligned}
-\Delta u & =w_{+}^{q}+\alpha g & & \text { in } \Omega \\
u & =0 & & \text { on } \partial \Omega .
\end{aligned}\right.
$$

It is clear that if $v$ is a fixed point of $T$, then $(u, v)$ solves the system (1.1). Thus we just have to show that $T$ has a fixed point in $E$, this will be achieved in several steps.

In what follows we denote by $C_{1}, C_{2}, \ldots$, any positive constants that depend only on the datum of the problem and that can be changed from one line to next one.

Step I : $T$ is well defined. Let $w \in E$, by using Sobolev's inequality we conclude that $w \in L^{r^{*}}(\Omega)$. Since $q \sigma<r^{*}$, then $w_{+}^{q} \in L^{\sigma}(\Omega) \subset L^{1}(\Omega)$. Hence, by Theorem 3.3, $u$ is well defined as the unique weak solution to the problem (4.6) and $u \in W_{0}^{1, \theta}(\Omega)$ for all $\theta<\frac{N}{N-1}$. Taking into consideration the hypothesis on $g$, we reach that $\left(w_{+}^{q}+\alpha g\right) \in L^{\sigma}(\Omega)$. Therefore, by Theorem 3.4, there exists $C_{1}$ such that

$$
\|\nabla u\|_{L^{\beta}(\Omega)} \leq C_{1}\left\|w_{+}^{q}+\alpha g\right\|_{L^{\sigma}(\Omega)}, \text { for all } \beta \leq \sigma^{*}=\frac{\sigma N}{N-\sigma} .
$$

Notice that by (4.2), we have $q \sigma<r^{*}$, hence using Hölder and Sobolev's inequalities, we get

$$
\|\nabla u\|_{L^{\beta}(\Omega)} \leq C_{2}\left(\|\nabla w\|_{L^{r}(\Omega)}^{q}+\alpha\|g\|_{L^{\sigma}(\Omega)}\right) \text {, for all } \beta \leq \sigma^{*} .
$$

From (2.2), $\sigma^{*}>p$, then (4.7) holds with $\beta=p$. Thus

$$
\|\nabla u\|_{L^{p}(\Omega)} \leq C_{2}\|\nabla w\|_{L^{r}(\Omega)}^{q}+C_{3}\|g\|_{L^{\sigma}(\Omega)} .
$$

Since $f \in L^{m}(\Omega)$ and $|\nabla u|^{p} \in L^{1}(\Omega)$ then $|\nabla u|^{p}+\lambda f \in L^{1}(\Omega)$ and $v$ is the unique weak solution to the problem (4.5). By applying again Theorem 3.3, $v \in W_{0}^{1, \theta}(\Omega)$ for all $\theta<\frac{N}{N-1}$ and then $T$ is well defined.

Step II : $T(E) \subset E$. First, by hypothesis $p m<\sigma^{*}$, we have $|\nabla u|^{p} \in L^{m}(\Omega)$. Second, by using the fact that $f \in L^{m}(\Omega)$ and by Theorem 3.4, we obtain that for all $\theta \leq m^{*}=\frac{m N}{N-m}$,

$$
\begin{aligned}
\|\nabla v\|_{L^{\theta}(\Omega)} & \leq C_{4}\left\||\nabla u|^{p}+\lambda f\right\|_{L^{m}(\Omega)} \\
& \leq C_{4}\left(\|\nabla u\|_{L^{p m}(\Omega)}^{p}+\lambda\|f\|_{L^{m}(\Omega)}\right) .
\end{aligned}
$$

Recalling again that $p m<\sigma^{*}$, then by using (4.7) with $\beta=p m$, we get

$$
\|\nabla u\|_{L^{p m}(\Omega)}^{p} \leq C_{5}\left(\|\nabla w\|_{L^{r}(\Omega)}^{p q}+\alpha^{p}\|g\|_{L^{\sigma}(\Omega)}^{p}\right) .
$$

Going back to (4.9), we conclude that

$$
\|\nabla v\|_{L^{\theta}(\Omega)} \leq C_{6}\left(\|\nabla w\|_{L^{r}(\Omega)}^{p q}+\alpha^{p}\|g\|_{L^{\sigma}(\Omega)}^{p}+\lambda\|f\|_{L^{m}(\Omega)}\right) .
$$


Since $r<\frac{m N}{N-m}$, by choosing $\theta=r$ in the previous inequality it holds that

$$
\|\nabla v\|_{L^{r}(\Omega)} \leq C_{7}\left(\|\nabla w\|_{L^{r}(\Omega)}^{p q}+\alpha^{p}\|g\|_{L^{\sigma}(\Omega)}^{p}+\lambda\|f\|_{L^{m}(\Omega)}\right) .
$$

Recall that $w \in E$, thus

$$
\|\nabla v\|_{L^{r}(\Omega)} \leq C_{8}\left(\ell+\alpha^{p}|| g\left\|_{L^{\sigma}(\Omega)}^{p}+\lambda\right\| f \|_{L^{m}(\Omega)}\right) .
$$

By choosing $\tilde{C}=C_{8}$ and taking into consideration the definition of $\ell$, we conclude that $\|\nabla v\|_{L^{r}(\Omega)} \leq \ell^{\frac{1}{p q}}$. Thus $v \in E$ and then $T(E) \subset E$.

Step III : $T$ is a continuous and compact operator on $E$ endowed with the topology of $W_{0}^{1,1}(\Omega)$.

- First, let us begin by proving the continuity of $T$. Assume that $\left\{w_{n}\right\}_{n} \subset E, w \in E$ are such that $w_{n} \rightarrow w$ in $W_{0}^{1,1}(\Omega)$ and define $v_{n}=T\left(w_{n}\right), v=T(w)$. Then $\left(u_{n}, v_{n}\right)$ and $(u, v)$ satisfy

$$
\left\{\begin{aligned}
-\Delta u_{n} & =w_{n+}^{q}+\alpha g & & \text { in } \Omega, \\
-\Delta u & =w_{+}^{q}+\alpha g & & \text { in } \Omega, \\
u_{n} & =u=0 & & \text { on } \partial \Omega
\end{aligned}\right.
$$

and

$$
\left\{\begin{aligned}
-\Delta v_{n} & =\left|\nabla u_{n}\right|^{p}+\lambda f & & \text { in } \Omega, \\
-\Delta v & =|\nabla u|^{p}+\lambda f & & \text { in } \Omega, \\
v_{n} & =v=0 & & \text { on } \partial \Omega .
\end{aligned}\right.
$$

Thanks to Sobolev's inequality in the space $W_{0}^{1,1}(\Omega)$, we have

$$
\left\|w_{n}-w\right\|_{L^{\frac{N}{N-1}(\Omega)}} \leq S_{1}\left\|w_{n}-w\right\|_{W_{0}^{1,1}(\Omega)} \rightarrow 0 \text { as } n \rightarrow \infty .
$$

Thus $w_{n} \rightarrow w$ strongly in $L^{s}(\Omega)$, for all $s \leq \frac{N}{N-1}$. Since $\left\{w_{n}\right\}_{n}$ is bounded in the space $W_{0}^{1, r}(\Omega)$, then using Vitali's theorem it follows that $w_{n} \rightarrow w$ strongly in $L^{a}(\Omega)$ for all $a<r^{*}$. In particular $w_{n+}^{q} \rightarrow w_{+}^{q}$ strongly in $L^{1}(\Omega)$. Therefore thanks to Theorem 3.3 we conclude that $u_{n} \rightarrow u$ strongly in $W_{0}^{1, a}(\Omega)$ for all $a<\frac{N}{N-2}$. In particular we have

$$
\left\|\nabla u_{n}-\nabla u\right\|_{L^{1}(\Omega)} \rightarrow 0 \text { as } n \rightarrow \infty .
$$

Now, by (4.10) it follows that

$$
\left\|\nabla u_{n}\right\|_{L^{p m}(\Omega)}^{p} \leq C\left(\left\|\nabla w_{n}\right\|_{L^{r}(\Omega)}^{p q}+\alpha^{p}\|g\|_{L^{\sigma}(\Omega)}^{p}\right) \leq C\left(\ell+\alpha^{p}\|g\|_{L^{\sigma}(\Omega)}^{p}\right) .
$$

Thus $\left\{u_{n}\right\}_{n}$ is bounded in $W_{0}^{1, p m}(\Omega)$. Using again Vitali's theorem we obtain that

$$
\left\|\nabla u_{n}-\nabla u\right\|_{L^{p}(\Omega)} \rightarrow 0 \text { as } n \rightarrow \infty .
$$

Hence by Theorem 3.3 there results that $v_{n} \rightarrow v$ strongly in $W_{0}^{1,1}(\Omega)$ and then the continuity of $T$ follows.

- Second, we show that $T$ is a compact operator. 
Let $\left\{w_{n}\right\}_{n} \subset E$ be such that $\left\|w_{n}\right\|_{W_{0}^{1,1}(\Omega)} \leq C$ and let $v_{n}=T\left(w_{n}\right)$. Since $\left\{w_{n}\right\}_{n} \subset E$, $\left\|\nabla w_{n}\right\|_{L^{r}(\Omega)} \leq C$ and then, up to a subsequence again denoted by $\left\{w_{n}\right\}_{n}$, we have

$$
w_{n} \rightarrow w \text { weakly in } W_{0}^{1, r}(\Omega) .
$$

By Rellich-Kondrachov's Theorem, it follows that $w_{n} \rightarrow w$ strongly in $L^{a}(\Omega)$ for all $a<r^{*}$. In particular, $w_{n+}^{q} \rightarrow w_{+}^{q}$ strongly in $L^{1}(\Omega)$.

Let $u$ to be the unique weak solution to the problem

$$
\left\{\begin{aligned}
-\Delta u & =w_{+}^{q}+\alpha g & & \text { in } \Omega \\
u & =0 & & \text { on } \partial \Omega .
\end{aligned}\right.
$$

By the result of Theorem 3.3, we reach that $\left\|\nabla u_{n}-\nabla u\right\|_{L^{1}(\Omega)} \rightarrow 0$ as $n \rightarrow \infty$. Now, setting $v=T(w)$ and following the same argument as in the proof of the continuity of $T$, we obtain that $v_{n} \rightarrow v$ strongly in $W_{0}^{1,1}(\Omega)$. Hence $T$ is compact. Therefore, by the Schauder Fixed Point Theorem we get the existence of $v \in E$ such that $T(v)=v$. It is clear that $v \in W_{0}^{1, r}(\Omega)$. Taking into consideration the hypothesis (4.2), it holds that $v \in W_{0}^{1, \rho}(\Omega)$ for all $\rho<\frac{m N}{N-m}$. Now going back to (4.6), we conclude that $u \in W_{0}^{1, \theta}(\Omega)$ for all $\theta<\frac{\sigma N}{N-\sigma}$. Thus the proof of Theorem 2.2 follows.

4.2. Proof of Theorem 2.3. Let $\alpha>0, \lambda>0, f \supsetneqq 0, g \supsetneqq 0$. Assume that the system

$$
\left\{\begin{aligned}
-\Delta u & =v^{q}+\alpha g & & \text { in } \Omega, \\
-\Delta v & =|\nabla u|^{p}+\lambda f & & \text { in } \Omega, \\
u=v & =0 & & \text { on } \partial \Omega, \\
u, v & \geq 0 & & \text { in } \Omega,
\end{aligned}\right.
$$

has a nonnegative solution $(u, v)$. Recall that $f, g \in L^{1}(\Omega)$.

In order to prove the existence of $\alpha^{*}$ and $\lambda^{*}$, we will distinguish two cases : $p, q>1$ and $p>1, q=1$.

In what follows we will use Young's inequality under this form

$$
\forall(a, b) \in(0,+\infty)^{2} \text { and } \forall s \in(1,+\infty), \quad a b \leq a^{s}+C_{s} b^{s^{\prime}} \text { where } C_{s}=\frac{s-1}{s^{s^{\prime}}} \text { and } s^{\prime}=\frac{s}{s-1} .
$$

- Case $p, q>1$.

Let $\phi \in \mathcal{C}_{0}^{\infty}(\Omega)$ such $\phi \supsetneqq 0$. Multiplying both equations of (4.15) by $\phi$ and integrating over $\Omega$, we obtain

$$
\int_{\Omega}\left(v^{q}+\alpha g\right) \phi=\int_{\Omega} \nabla u \nabla \phi
$$

and

$$
\int_{\Omega}\left(|\nabla u|^{p}+\lambda f\right) \phi=-\int_{\Omega} \phi \Delta v=\int_{\Omega} v(-\Delta \phi) .
$$

Determination of $\alpha^{*}$.

Thanks to Young's inequality, (4.16) yields

$$
\int_{\Omega}\left(v^{q}+\alpha g\right) \phi \leq \int_{\Omega} \phi|\nabla u|^{p}+C_{p} \int_{\Omega} \phi^{1-p^{\prime}}|\nabla \phi|^{p^{\prime}}
$$


By the nonnegativity of each term of (4.17) we get

$$
\int_{\Omega}|\nabla u|^{p} \phi \leq \int_{\Omega} v(-\Delta \phi)
$$

Therefore Young's inequality yields

$$
\int_{\Omega}|\nabla u|^{p} \phi \leq \int_{\Omega} v^{q} \phi+C_{q} \int_{\Omega} \phi^{1-q^{\prime}}|\Delta \phi|^{q^{\prime}}
$$

Let us set

$$
F(\phi)=C_{p} \int_{\Omega} \phi^{1-p^{\prime}}|\nabla \phi|^{p^{\prime}}+C_{q} \int_{\Omega} \phi^{1-q^{\prime}}|\Delta \phi|^{q^{\prime}} .
$$

From (4.18) and (4.20), we deduce

$$
\alpha \int_{\Omega} g \phi \leq F(\phi)
$$

and then $\alpha \leq \alpha^{*}$ with

$$
\alpha^{*}:=\inf \left\{F(\phi) ; 0 \leq \phi \in \mathcal{C}_{0}^{\infty}(\Omega) \text { and } \int_{\Omega} g \phi=1\right\} .
$$

To end this part we have to show that $\alpha^{*}<\infty$.

It is clear that $\alpha^{*} \geq 0$. Let $\psi \in \mathcal{C}_{0}^{\infty}(\Omega)$ be such that $\psi \geq 0$ and define $\phi=\bar{C} \psi^{\theta}$ with $\theta=$ $\left[\max \left\{p^{\prime}, 2 q^{\prime}\right\}\right]+1$ (where [.] denotes the integer part) and $\overline{\bar{C}}>0$ is chosen such that $\int_{\Omega} g \phi=1$. Since $\theta \in \mathbb{N}^{*}, \phi \in \mathcal{C}_{0}^{\infty}(\Omega)$. Moreover

$$
\phi^{1-p^{\prime}}|\nabla \phi|^{p^{\prime}}=\bar{C} \theta^{p^{\prime}} \psi^{\theta-p^{\prime}}|\nabla \psi|^{p^{\prime}}
$$

and

Thus

$$
\phi^{1-q^{\prime}}|\Delta \phi|^{q^{\prime}} \leq C(q, \theta, \bar{C})\left(\psi^{\theta-q^{\prime}}|\Delta \psi|^{q^{\prime}}+\psi^{\theta-2 q^{\prime}}|\nabla \psi|^{2 p^{\prime}}\right) .
$$

$$
F(\phi) \leq \theta^{p^{\prime}} \int_{\Omega} \psi^{\theta-p^{\prime}}|\nabla \psi|^{p^{\prime}} d x+C(q, \theta, \bar{C}) \int_{\Omega}\left(\psi^{\theta-q^{\prime}}|\Delta \psi|^{q^{\prime}}+\psi^{\theta-2 q^{\prime}}|\nabla \psi|^{2 p^{\prime}}\right) d x<\infty .
$$

Hence $\alpha^{*}<\infty$.

It is clear that if $\alpha>\alpha^{*}$, system (1.1) does not have any positive solution.

Determination of $\lambda^{*}$.

For this, we will proceed in the same way as before.

By applying Young's inequality, (4.17) implies

$$
\int_{\Omega}\left(|\nabla u|^{p}+\lambda f\right) \phi \leq \int_{\Omega} \phi v^{q}+C_{q} \int_{\Omega} \phi^{1-q^{\prime}}|\Delta \phi|^{q^{\prime}}
$$

Thanks to the nonnegativity of each term, (4.16) yields

$$
\int_{\Omega} v^{q} \phi \leq \int_{\Omega} \nabla u \nabla \phi
$$

and then

$$
\int_{\Omega} v^{q} \phi \leq \int_{\Omega} \phi|\nabla u|^{p}+C_{p} \int_{\Omega} \phi^{1-p^{\prime}}|\nabla \phi|^{p^{\prime}}
$$


Therefore (4.23) and (4.25) imply

$$
\lambda \int_{\Omega} f \phi \leq F(\phi) .
$$

and then

$$
\lambda^{*}=\inf \left\{F(\phi) ; 0 \leq \phi \in \mathcal{C}_{0}^{\infty}(\Omega) \text { and } \int_{\Omega} f \phi=1\right\} .
$$

As above we can show that $\lambda^{*}<\infty$. Thus $\lambda \leq \lambda^{*}$.

- Case $q=1$ and $p>1$.

Let $\varphi \in \mathcal{C}_{0}^{\infty}(\Omega)$ be such that $\varphi \supsetneqq 0$ and define $\phi$ to be the unique solution to the following problem

$$
\left\{\begin{array}{rlll}
-\Delta \phi & = & \text { in } \Omega \\
\phi & = & 0 & \text { on } \partial \Omega .
\end{array}\right.
$$

It is clear that $\phi \in \mathcal{C}^{2}(\bar{\Omega})$ and $-\Delta \phi \supsetneqq 0$. Then multiplying the first equation in (1.1) by $-\Delta \phi$, the second equation by $\phi$ and integrating to obtain

$$
\int_{\Omega} v(-\Delta \phi)+\alpha \int_{\Omega} g(-\Delta \phi)=\int_{\Omega}(-\Delta u)(-\Delta \phi)=\int_{\Omega} \nabla u \cdot \nabla(-\Delta \phi)
$$

\section{Determination of $\alpha^{*}$.}

Thanks to Young's inequality, we have

$$
\int_{\Omega} v(-\Delta \phi)+\alpha \int_{\Omega} g(-\Delta \phi) \leq \int_{\Omega}|\nabla u|^{p} \phi+C_{p} \int_{\Omega} \phi^{1-p^{\prime}}|\nabla(-\Delta \phi)|^{p^{\prime}} .
$$

But

$$
\int_{\Omega}|\nabla u|^{p} \phi \leq \int_{\Omega}|\nabla u|^{p} \phi+\lambda \int_{\Omega} f \phi=\int_{\Omega}(-\Delta v) \phi=\int_{\Omega} v(-\Delta \phi)
$$

Therefore

$$
\int_{\Omega} v(-\Delta \phi)+\alpha \int_{\Omega} g(-\Delta \phi) \leq \int_{\Omega}(-\Delta v) \phi+C_{p} \int_{\Omega} \phi^{1-p^{\prime}}|\nabla(-\Delta \phi)|^{p^{\prime}} .
$$

Thus

$$
\alpha \int_{\Omega} g(-\Delta \phi) \leq C_{p} \int_{\Omega} \phi^{1-p^{\prime}}|\nabla(-\Delta \phi)|^{p^{\prime}}
$$

Setting $G(\varphi)=C_{p} \int_{\Omega} \phi^{1-p^{\prime}}|\nabla \varphi|^{p^{\prime}}$. Going back to the definition of $\phi$ and using the strong maximum principle, then there exists $C>0$ such that $\phi \geq C$ in the support of $\varphi$. Thus $G(\varphi)$ is well defined for all $\varphi \in \mathcal{C}_{0}^{\infty}(\Omega)$ and $\varphi \supsetneqq 0$.

Define

$$
\alpha^{*}:=\inf \left\{G(\varphi) \mid \varphi \in \mathcal{C}_{0}^{\infty}(\Omega), \varphi \supsetneqq 0 \text { and } \int_{\Omega} g \varphi=1\right\},
$$

then $\alpha^{*}<\infty$ and $\alpha \leq \alpha^{*}$.

Determination of $\lambda^{*}$.

In the same way, we obtain 


$$
\int_{\Omega}|\nabla u|^{p} \phi+\lambda \int_{\Omega} f \phi=\int_{\Omega}(-\Delta v) \phi=\int_{\Omega} v(-\Delta \phi)
$$

and

$$
\int_{\Omega} v(-\Delta \phi) \leq \int_{\Omega}|\nabla u|^{p} \phi+C_{p} \int_{\Omega} \phi^{1-p^{\prime}}|\nabla(-\Delta \phi)|^{p^{\prime}}
$$

Thus

$$
\lambda \int_{\Omega} f \phi \leq G(\varphi)
$$

and then $\lambda \leq \lambda^{*}$ with

$$
\lambda^{*}=\inf \left\{G(\varphi) \mid \int_{\Omega} f \phi=1\right\} .
$$

We conclude as above that $\lambda^{*}<\infty$.

\section{Remark 3.}

(1) Consider the set

$\Sigma:=\{(\lambda, \alpha) \in(0,+\infty) \times(0,+\infty) \mid$ such that the system $(1.1)$ has a positive solution $\}$.

It is clear that under the hypotheses of Theorem 2.2, we have $\Sigma \neq \emptyset$. Hence setting

$$
\begin{aligned}
& \hat{\lambda}=\max \{\lambda>0 \text { such that }(\lambda, \alpha) \in \Sigma \text { for some } \alpha>0\}, \\
& \hat{\alpha}=\max \{\alpha>0 \text { such that }(\lambda, \alpha) \in \Sigma \text { for some } \lambda>0\} .
\end{aligned}
$$

It follows that $\hat{\lambda}, \hat{\alpha}>0$. Going back to the proof of Theorem 2.3, we obtain that $\lambda^{*} \geq \hat{\lambda}>0$ and $\alpha^{*} \geq \hat{\alpha}>0$.

(2) Under additional hypotheses on $f$ and $g$, we get some explicit bounds from below for $\alpha^{*}$ and $\lambda^{*}$ defined in the proof of Theorem 2.3.

The first case : $p, q>1$ : Assume that $f, g \in L^{\frac{N}{p^{\prime}}}(\Omega)$ and let $\phi \in \mathcal{C}_{0}^{\infty}(\Omega)$, then

$$
F(\phi) \geq C_{p} \int_{\Omega} \phi^{1-p^{\prime}}|\nabla \phi|^{p^{\prime}}=C_{p}\left(p^{\prime}\right)^{p^{\prime}} \int_{\Omega}\left|\nabla \phi^{\frac{1}{p^{\prime}}}\right|^{p^{\prime}} .
$$

Without loss of generality we can assume that $p^{\prime}<N$. By using Sobolev's inequality we reach that

$$
F(\phi) \geq C_{p}\left(\int_{\Omega}|\phi|^{\frac{N}{N-p^{\prime}}}\right)^{\frac{N-p^{\prime}}{N}} .
$$

Applying Hölder's inequality, we obtain

$$
\int_{\Omega} f \phi \leq\|f\|_{L^{\frac{N}{p^{\prime}}}(\Omega)}\|\phi\|_{L^{\frac{N}{N-p^{\prime}}}(\Omega)} .
$$

Thus

$$
\frac{F(\phi)}{\int_{\Omega} f \phi} \geq \frac{C_{p}}{\|f\|_{L^{\frac{N}{p^{\prime}}}(\Omega)}} .
$$


Therefore it holds that $\lambda^{*} \geq \frac{C_{p}}{\|f\|_{L^{\frac{N}{p^{\prime}}}(\Omega)}}$. In the same way we reach that $\alpha^{*} \geq \frac{C_{p}}{\|g\|_{L^{\frac{N}{p^{\prime}}}(\Omega)}}$.

The second case: $q=1$ and $p>1$ : Assume that $f, g \in L^{\frac{\theta N}{(\theta-1) N+\theta}}(\Omega)$ with $\theta$ is chosen such that $1<\theta<\min \left\{\frac{N}{\left(N-3\left(p^{\prime}-1\right)\right)_{+}}, p^{\prime}\right\}$. It is clear that $\frac{\left(p^{\prime}-1\right) \theta}{p^{\prime}-\theta} \leq \frac{\theta N}{N-3 \theta}$.

Recall that $\phi$ is the unique solution of problem (4.28). Taking into consideration the definition of $\phi$ and $\theta$, and by Theorem 3.4, it follows that

$$
\|\phi\|_{L^{\frac{\left(p^{\prime}-1\right) \theta}{p^{\prime}-1}}(\Omega)} \leq C\|\varphi\|_{L^{\theta^{*}}(\Omega)} \leq C\|\nabla \varphi\|_{L^{\theta}(\Omega)} .
$$

Using Hölder's inequality, it follows that

$$
\begin{aligned}
\int_{\Omega}|\nabla \varphi|^{\theta} & =\int_{\Omega}|\nabla \varphi|^{\theta} \phi^{\frac{\left(1-p^{\prime}\right) \theta}{p^{\prime}}} \phi^{\frac{\left(p^{\prime}-1\right) \theta}{p^{\prime}}} \leq\left(\int_{\Omega} \phi^{1-p^{\prime}}|\nabla \varphi|^{p^{\prime}}\right)^{\frac{\theta}{p^{\prime}}}\left(\int_{\Omega}|\varphi|^{\frac{\left(p^{\prime}-1\right) \theta}{p^{\prime}-\theta}}\right)^{\frac{p^{\prime}-\theta}{p^{\prime}}} \\
& \leq C\left(\int_{\Omega} \phi^{1-p^{\prime}}|\nabla \varphi|^{p^{\prime}}\right)^{\frac{\theta}{p^{\prime}}}\left(\int_{\Omega}|\varphi|^{\theta^{*}}\right)^{\frac{\left(p^{\prime}-1\right) \theta}{p^{\prime} \theta^{*}}}
\end{aligned}
$$

Thus, using Sobolev's inequality it holds that

$$
\left(\int_{\Omega}|\nabla \varphi|^{\theta}\right)^{\frac{1}{\theta}} \leq C \int_{\Omega} \phi^{1-p^{\prime}}|\nabla \varphi|^{p^{\prime}}=C G(\varphi) .
$$

On the other hand we have

$$
\int_{\Omega} f \phi \leq\|f\|_{L^{\frac{\theta N}{(\theta-1) N+\theta}(\Omega)}}\|\phi\|_{L^{\theta^{*}}(\Omega)} .
$$

Hence

$$
\frac{G(\varphi)}{\int_{\Omega} f \varphi} \geq \frac{C(p, \Omega)\left(\int_{\Omega}|\nabla \varphi|^{\theta}\right)^{\frac{1}{\theta}}}{\int_{\Omega} f \varphi} \geq \frac{C(p, \Omega)}{\|f\|_{L^{\frac{\theta N}{(\theta-1) N+\theta}}(\Omega)}} .
$$

Thus $\lambda^{*} \geq \frac{C(p, \Omega)}{\|f\|_{L^{\frac{\theta N}{(\theta-1) N+\theta}}(\Omega)}}$.

In the same way we obtain that $\alpha^{*} \geq \frac{C(p, \Omega)}{\|g\|_{L^{\frac{\theta N}{(\theta-1) N+\theta}}(\Omega)}}$.

Remark 4. The existence result in Theorem 2.2 is obtained under the hypothesis that $p q>1$ and smallness condition on $(\alpha, \lambda)$. It will be interesting to show that nonexistence result, for large values of $(\alpha, \lambda)$, obtained in Theorem 2.3 holds under the condition $p q>1$. 
4.3. Proof of Theorem 2.4. In this case we take $q=1$ and $g=0$ in Theorem 2.2. Since $f \in L^{m}(\Omega)$ with $m>\max \left\{1, \frac{N}{3 p^{\prime}}\right\}$, we can fix $m_{0}$ such that $m_{0} \leq m$ and $\frac{N}{3 p^{\prime}}<m_{0}<N$.

If $m_{0} \geq \frac{N}{2}$, we take $\sigma_{0}=N-\varepsilon$ with $\varepsilon$ small enough, however, if $\frac{N}{3 p^{\prime}}<m_{0}<\frac{N}{2}$, we choose $\sigma_{0}=\frac{3 p m_{0}}{p+2}$. Hence in all cases, the condition (2.2) holds and then by Theorem 2.2, we get the existence of $(u, v) \in W_{0}^{1, \rho}(\Omega) \times W_{0}^{1, \theta}(\Omega)$, for all $\rho<\frac{m_{0} N}{N-m_{0}}, \theta<\frac{\sigma_{0} N}{N-\sigma_{0}}$, such that $(u, v)$ solves the system

$$
\left\{\begin{aligned}
-\Delta u & =v & & \text { in } \Omega, \\
-\Delta v & =|\nabla u|^{p}+\lambda f & & \text { in } \Omega, \\
u=v & =0 & & \text { on } \partial \Omega, \\
u, v & \geq 0 & & \text { in } \Omega .
\end{aligned}\right.
$$

It is clear that:

$$
u=\Delta u=0 \quad \text { on } \partial \Omega \quad \text { and } \quad \Delta^{2} u=-\Delta v=|\nabla u|^{p}+\lambda f \quad \text { in } \Omega .
$$

Hence $u$ solves (4.39). Now using the regularity results in [19], we reach that $u \in \mathcal{C}^{s}(\Omega)$ for all $s<4-\frac{N}{m}$, in particular $u \in \mathcal{C}^{s}(\Omega)$ for all $s<2$ if $m \geq \frac{N}{2}$ and $u \in W^{4, m}(\Omega)$ if $m<\frac{N}{2}$. Hence we conclude.

Remark 5. Taking into consideration the nonlinear nature of the above arguments used in the proof of Theorem 2.2, then we can extend our existence result for more general operator like the $m$-laplacian operator where $\Delta_{m}(u):=\operatorname{div}\left(|\nabla u|^{m-2} \nabla u\right)$. More precisely if we consider the system

$$
\left\{\begin{aligned}
-\Delta_{m_{1}} u & =v^{q}+\alpha g & & \text { in } \Omega, \\
-\Delta_{m_{2}} v & =|\nabla u|^{p}+\lambda f & & \text { in } \Omega, \\
u=v & =0 & & \text { on } \partial \Omega, \\
u, v & \geq 0 & & \text { in } \Omega,
\end{aligned}\right.
$$

with $m_{1}, m_{2}>2-\frac{1}{N}$, then if $f \in L^{\theta}(\Omega)$ and $g \in L^{\sigma}(\Omega)$, where $(\theta, \sigma)$ satisfies

$$
\left\{\begin{aligned}
\theta, \sigma & \in(1, N) \\
p \theta & <\frac{\sigma N\left(m_{1}-1\right)}{N-\sigma}, \\
\frac{q \sigma N}{N+q \sigma} & <\frac{\theta N\left(m_{2}-1\right)}{N-\theta},
\end{aligned}\right.
$$

we can show the existence of a bounded set $\bar{\Pi} \subset \mathbb{R}^{+} \times \mathbb{R}^{+}$defined by

$$
\bar{\Pi} \equiv\left\{(\lambda, \alpha) \in\left(\mathbb{R}^{+}\right)^{2}: \lambda\|f\|_{L^{\theta}(\Omega)}+\alpha^{p}\|g\|_{L^{\sigma}(\Omega)}^{p} \leq \bar{\Lambda}\right\}
$$

such that for all $(\lambda, \alpha) \in \bar{\Pi}$, the system (4.37) has a positive solution $(u, v)$ with $(u, v) \in W_{0}^{1, \rho}(\Omega) \times$ $W_{0}^{1, r}(\Omega)$ for all $\rho<\frac{\sigma N\left(m_{1}-1\right)}{N-\sigma}$ and $r<\frac{\theta N\left(m_{2}-1\right)}{N-\theta}$. 
4.4. Some nonexistence results. In this subsection we will show that the regularity conditions (2.2) are optimal for existence at least in some explicit cases. More precisely we will analyze the problem

$$
\left\{\begin{aligned}
\Delta^{2} u & =|\nabla u|^{p}+\lambda f & & \text { in } \Omega, \\
u=\Delta u & =0 & & \text { on } \partial \Omega, \\
u & \geq 0 & & \text { in } \Omega .
\end{aligned}\right.
$$

that is the case where $q=1$ and $g \equiv 0$.

Let us begin by proving the following result.

Theorem 4.1. Assume that $0 \supsetneqq f \in L^{m}(\Omega)$ with $m \geq 1$ and $p>1$. Define

$$
\Lambda(f) \equiv \inf _{\phi \in \mathcal{C}_{0}^{\infty}(\Omega), \phi \neq 0} \frac{\int_{\Omega} \frac{\left|\nabla\left(|\phi|^{p^{\prime}-2} \phi(-\Delta \phi)\right)\right|^{p^{\prime}}}{|\phi|^{\frac{p}{(p-1)^{2}}}} d x}{\int_{\Omega} f|\phi|^{p^{\prime}} d x} .
$$

Assume that Problem (4.39) has a positive solution for some $\lambda>0$, then $\Lambda(f)>0$.

Proof : We follow closely the arguments used in [5]. Suppose that Problem (4.39) has positive solution $u$. Setting $v=-\Delta u$, then $v$ solves

$$
\left\{\begin{aligned}
-\Delta v & =|\nabla u|^{p}+\lambda f & & \text { in } \Omega, \\
v & =0 & & \text { on } \partial \Omega
\end{aligned}\right.
$$

Thus $v>0$, hence $-\Delta u>0$ in $\Omega$. Let $\phi \in \mathcal{C}_{0}^{\infty}(\Omega)$ such $\phi \neq 0$. Using $|\phi|^{p^{\prime}}$ as a test function in (4.39), we obtain

$$
\int_{\Omega}(-\Delta u)\left(-\Delta|\phi|^{p^{\prime}}\right) d x=\int_{\Omega}|\nabla u|^{p}|\phi|^{p^{\prime}} d x+\lambda \int_{\Omega} f|\phi|^{p^{\prime}} d x .
$$

Since $p^{\prime}>1$, then using Kato's inequality we reach that

$$
-\Delta|\phi|^{p^{\prime}} \leq p^{\prime}|\phi|^{p^{\prime}-2} \phi(-\Delta \phi) .
$$

Since $-\Delta u \geq 0$, using Young's inequality, it follows that

$$
\begin{aligned}
\int_{\Omega}(-\Delta u)\left(-\Delta|\phi|^{p^{\prime}}\right) d x & \leq p^{\prime} \int_{\Omega}|\phi|^{p^{\prime}-2} \phi(-\Delta \phi)(-\Delta u) d x \\
& \leq p^{\prime} \int_{\Omega} \nabla u \nabla\left(|\phi|^{p^{\prime}-2} \phi(-\Delta \phi)\right) d x \\
& \leq \varepsilon \int_{\Omega}|\nabla u|^{p}|\phi|^{p^{\prime}} d x+C(\varepsilon) \int_{\Omega} \frac{\left|\nabla\left(|\phi|^{p^{\prime}-2} \phi(-\Delta \phi)\right)\right|^{p^{\prime}}}{|\phi|^{\frac{p}{(p-1)^{2}}}} d x .
\end{aligned}
$$

Choosing $p^{\prime} \varepsilon \leq 1$ and going back to (4.41), we conclude that

$$
\lambda \int_{\Omega} f|\phi|^{p^{\prime}} d x \leq C(\varepsilon, p) \int_{\Omega} \frac{\left|\nabla\left(|\phi|^{p^{\prime}-2} \phi(-\Delta \phi)\right)\right|^{p^{\prime}}}{|\phi|^{\frac{p}{(p-1)^{2}}}} d x .
$$


Thus

$$
\lambda \leq C(\varepsilon, p) \frac{\int_{\Omega} \frac{\left|\nabla\left(|\phi|^{p^{\prime}-2} \phi(-\Delta \phi)\right)\right|^{p^{\prime}}}{|\phi|^{\frac{p}{(p-1)^{2}}}} d x}{\int_{\Omega} f|\phi|^{p^{\prime}} d x}
$$

and the result follows.

Remark 6. Let $\phi \in \mathcal{C}_{0}^{\infty}(\Omega)$ and consider the operator $Q$ defined

$$
Q(\phi)=\frac{\int_{\Omega} \frac{\left|\nabla\left(|\phi|^{p^{\prime}-2} \phi(-\Delta \phi)\right)\right|^{p^{\prime}}}{|\phi|^{\frac{p}{(p-1)^{2}}}} d x}{\int_{\Omega} f|\phi|^{p^{\prime}} d x},
$$

then $Q(\alpha \phi)=Q(\phi)$ for all $\alpha \in \mathbb{R}^{*}$.

As a direct consequence of Theorem 4.1 we can show that Problem (4.39) has non solution if $\lambda$ is large. This follows directly by estimate (4.42).

We are now able to prove the next nonexistence result.

Theorem 4.2. Assume that $1 \leq m<\max \left\{1, \frac{N}{3 p^{\prime}}\right\}$ where $p>1$, then there exists $f \in L^{m}(\Omega)$ with $f \gtrless 0$ such that Problem (4.39) does not have any positive solution for any $\lambda>0$.

Proof : Without loss of generality we can assume that $N>p^{\prime}$. Assume that $\Omega=B_{1}(0) \subset \mathbb{R}^{N}$ and fix $m<\frac{N}{3 p^{\prime}}$. Consider the function $f(x)=\frac{1}{|x|^{3 p^{\prime}+\varepsilon}}$. Then we can choose $\varepsilon$ small enough such that $f \in L^{m}\left(B_{1}(0)\right)$. In order to show the nonexistence result we will prove that $\Lambda(f)=0$ and then we will conclude by Theorem 4.1.

Let $\phi$ be the function defined by

$$
\phi(x)=\left\{\begin{array}{lll}
\frac{1}{|x|^{\theta}} & \text { if } & |x| \leq \frac{1}{4} \\
(1-|x|)^{\gamma} & \text { if } \quad \frac{1}{2} \leq|x| \leq 1,
\end{array}\right.
$$

where $\theta=\frac{N-\left(3 p^{\prime}+\varepsilon\right)}{p^{\prime}}, \gamma>\frac{3 p^{\prime}-1}{p^{\prime}}$. It is clear that $\phi \in \mathcal{C}^{2}\left(B_{1}(0) \backslash\{0\}\right)$ and $\phi>0$ in $B_{1}(0)$. Moreover, we have

$$
\int_{B_{1}(0)} \frac{\left|\nabla\left(|\phi|^{p^{\prime}-2} \phi(-\Delta \phi)\right)\right|^{p^{\prime}}}{|\phi|^{\frac{p}{(p-1)^{2}}}} d x=\int_{B_{\frac{1}{4}}(0)}+\int_{\frac{1}{4}<|x|<\frac{1}{2}}+\int_{\frac{1}{2}<|x|<1} .
$$

Since $\phi>0$ for $\frac{1}{4}<|x|<\frac{1}{2}$, then $\int_{\frac{1}{4}<|x|<\frac{1}{2}}=C<\infty$.

Now by a direct computation we reach that

$$
\int_{B_{\frac{1}{4}}(0)} \frac{\left|\nabla\left(|\phi|^{p^{\prime}-2} \phi(-\Delta \phi)\right)\right|^{p^{\prime}}}{|\phi|^{\frac{p}{(p-1)^{2}}}} d x \leq C \int_{B_{\frac{1}{4}}(0)} \frac{1}{|x|^{p^{\prime}(\theta+3)}} d x .
$$


Since $\theta=\frac{N-\left(3 p^{\prime}+\varepsilon\right)}{p^{\prime}}$, then $p^{\prime}(\theta+3)=N-\varepsilon<N$. Thus

$$
\int_{B_{\frac{1}{4}}(0)} \frac{\left|\nabla\left(|\phi|^{p^{\prime}-2} \phi(-\Delta \phi)\right)\right|^{p^{\prime}}}{|\phi|^{\frac{p}{(p-1)^{2}}}} d x \leq C .
$$

We deal now with the integral $\int_{\frac{1}{2}<|x|<1}$. In this case we have

$$
\int_{\frac{1}{2}<|x|<1} \frac{\left|\nabla\left(|\phi|^{p^{\prime}-2} \phi(-\Delta \phi)\right)\right|^{p^{\prime}}}{|\phi|^{\frac{p}{(p-1)^{2}}}} d x \leq C \int_{\frac{1}{2}<|x|<1}(1-|x|)^{p^{\prime}(\gamma-3)} d x .
$$

Since $\gamma>\frac{3 p^{\prime}-1}{p^{\prime}}$, then $p^{\prime}(\gamma-3)>-1$. Therefore

$$
\int_{\frac{1}{2}<|x|<1} \frac{\left|\nabla\left(|\phi|^{p^{\prime}-2} \phi(-\Delta \phi)\right)\right|^{p^{\prime}}}{|\phi|^{\frac{p}{(p-1)^{2}}}} d x \leq C .
$$

As a conclusion we have proved that

$$
\int_{B_{1}(0)} \frac{\left|\nabla\left(|\phi|^{p^{\prime}-2} \phi(-\Delta \phi)\right)\right|^{p^{\prime}}}{|\phi|^{\frac{p}{(p-1)^{2}}}} d x<\infty .
$$

On the other hand we have $\int_{\Omega} f|\phi|^{p^{\prime}} d x=+\infty$. Hence $Q(\alpha \phi)=0$. Now using an approximation argument we obtain that $\Lambda(f)=0$ and therefore the result follows.

Remark 7. Combining the existence result in Theorem 2.4 and the result of Theorem 4.1 we obtain that if $0 \supsetneqq f \in L^{m}(\Omega)$ where $m>\max \left\{1, \frac{N}{3 p^{\prime}}\right\}$, then $\Lambda(f)>0$.

It will be nice if we can prove directly that if $f \in L^{m}(\Omega)$ with $m>\max \left\{1, \frac{N}{3 p^{\prime}}\right\}$, then $\Lambda(f)>0$.

Acknowledgments :

- The authors would like to thank Prof. Michel PIERRE for his helpful suggestions and fruitful discussions during the preparation of this work.

- Part of this work was realized while the first author was visiting the Institute Elie Cartan, Université Lorraine. He would like to thanks the institute for the warm hospitality.

\section{REFERENCES}

[1] B. Abdellaoui, A. Dall'Aglio, and I. Peral, Some remarks on elliptic problems with critical growth in the gradient. J. Differential Equations 222 (2006), 21-62.

[2] D. R. Adams, M. Pierre, Capacitary strong type estimates in semilinear problems. Ann. Inst. Fourier (Grenoble) 41 (1991), no. 1, 117-135.

[3] N.E. Alaa, M. Pierre, Weak solutions of some quasilinear elliptic equations with data measures. SIAM J. Math. Anal., 24, (1993), 23-35.

[4] A. Attar, R. Bentifour, Existence of positive solutions to nonlinear elliptic system involving gradient term and reaction potential . Electronic Journal of Differential Equations, 2017 (2017), no. 113, 1-10.

[5] P. Baras, M. Pierre, Critère d'existence des solutions positives pour des équations semi-linéaires non monotones, Ann. I.H.P. 2, (3) (1985), 185-212.

[6] P. Baras, M. Pierre, Singularités éliminables pour des équations semi-linéaires, Ann. Inst. Fourier 34, (1984), no. 1, 185-206. 
[7] G. Barles, A. Porretta, Uniqueness for unbounded solutions to stationary viscous Hamilton-Jacobi equations. Ann. Sc. Norm. Super. Pisa Cl. Sci. 5 (2006), no. 1, 107-136.

[8] L. Boccardo, G. Croce, Elliptic Partial Differential Equations : existence and regularity of distributional solutions. Studies in Mathematics 55 (2014), De Gruyter.

[9] L. Boccardo, T. Gallouët, F. Murat, A unified representation of two existence results for problems with natural growth, Research Notes in Mathematics 296 (1993), 127-137.

[10] L. Boccardo, F. Murat, J.-P. Puel, Existence des solutions non bornées pour certaines équations quasi-linéaires, Portugal Math. 41 (1982), 507-534

[11] L. Boccardo, L. Orsina, A. Porretta, Existence of finite energy solutions for elliptic systems with $L^{1}-$ value nonlinearities Mathematical Models and Methods in Applied Sciences 18, (2008), no.5, 669-687.

[12] L. Boccardo, L. Orsina, J.-P. Puel, A quasilinear elliptic system with natural growth terms. Annali di Matematica. 194, (2015), no.3, 1733-1750.

[13] H. Brézis, Functional analysis, Sobolev spaces and partial differential equations. Universitext. Springer, New York, 2011.

[14] K. Cho, H.J. Choe, Nonlinear degenerate elliptic partial differential equations with critical growth conditions on the gradient, Proc. A.M.S. 123, (1995), no 12, 3789-3796.

[15] S. Clain, J. Rappaz, M. Swierkosz, R. Touzani, Numerical modeling of induction heating for two dimentional geometrie, Math. Models Methods Appl. Sci. 3, (1993), no. 6, 805-822.

[16] J. I. Díaz, M. Lazzo, P.G. Schmidt, Large solutions for a system of elliptic equation arising from fluid dynamics. Siam Journal on Mathematical Analysis, 37 (2005), 490-513.

[17] C. Escudero, I. Peral, Some fourth order nonlinear elliptic problems related to epitaxial growth, J. Differential Equations, 254 (2013), 2515-2531.

[18] V. Ferone, F. Murat, Quasilinear problems having quadratic growth in the gradient : an existence result when the source term is small, Equations aux dérivées partielles et applications, 497-515, Gauthier-Villars, Ed. Sci. Méd. Elsevier, Paris, 1998.

[19] F. Gazzola, H.C. Grunau, G. Sweers, Polyharmonic boundary value problems. Positivity preserving and nonlinear higher order elliptic equations in bounded domains. Lecture Notes in Mathematics, 1991. Springer-Verlag, Berlin, 2010.

[20] N. Grenon, F. Murat, A. Porretta, Existence and a priori estimate for elliptic problems with subquadratic gradient dependent terms, C. R. Acad. Sci. Paris, Ser. I 342 (2006), 23-28.

[21] N. Grenon, C. Trombetti, Existence results for a class of nonlinear elliptic problems with p-growth in the gradient, Nonlinear Anal. 52 (2003), no.3, 931-942.

[22] K Hansson, V.G. Maz'ya, I.E. Verbitsky, Criteria of solvability for multidimensional Riccati equations, Ark. Mat., 37, (1999), 87-120.

[23] J. Leray and J.-L. Lions, Quelques résultats de Višik sur les problèmes elliptiques non linéaires par les méthodes de Minty-Browder, Bull. Soc. Math. France 93, (1965), 97-107.

[24] P.-L. Lions, Generalized solutions of Hamilton-Jacobi Equations, Pitman Res. Notes Math. 62 (1982).

[25] N.C. Phuc, Morrey global bounds and qusilinear Riccarti type equation bellow the natural exponent, J. math. Pures Appl. 102, (2014), 99-123.

B. Abdellaoui, A. Attar, Laboratoire d’Analyse Nonlinéaire et Mathématiques Appliquées. Département de Mathématiques, Université Abou Bakr Belkaïd, Tlemcen, Tlemcen 13000, Algeria.

EL-HAJ LAAMRI, Institut Elie CARTAN, UNIVERSITÉ LORRAINE,

B. P. 239, 54506 Vandeeuvre lés Nancy, France.

E-mail addresses:

boumediene.abdellaoui@inv.uam.es, ahm.attar@yahoo.fr, el-haj.laamri@univ-lorraine.fr. 